\title{
A Galerkin Finite Element Method for Two-Point Boundary Value Problems of Ordinary Differential Equations
}

\author{
Gentian Zavalani \\ Faculty of Mathematics and Physics Engineering Polytechnic University of Tirana, Albania
}

Email address:

zavalanigentian@hotmail.com

To cite this article:

Gentian Zavalani. A Galerkin Finite Element Method for Two-Point Boundary Value Problems of Ordinary Differential Equations. Applied and Computational Mathematics. Vol. 4, No. 2, 2015, pp. 64-68. doi: 10.11648/j.acm.20150402.15

\begin{abstract}
In this paper, we present a new method for solving two-point boundary value problem for certain ordinary differential equation. The two point boundary value problems have great importance in chemical engineering, deflection of beams etc. In this study, Galerkin finite element method is developed for inhomogeneous second-order ordinary differential equations. Several examples are solved to demonstrate the application of the finite element method. It is shown that the finite element method is simple, accurate and well behaved in the presence of singularities.
\end{abstract}

Keywords: Exact Solution, Two-Point Value Boundary Problem, Finite Element Method

\section{Introduction}

Two-point boundary-value problems in ordinary differential equations occur in many branches of physics; examples include the two-dimensional, incompressible, onedimensional heat transfer, boundary layer equations, etc. The corresponding ordinary differential equations can be nonlinear or linear but with complex coefficients. If the differential equation is nonlinear or linear but with complex coefficients, a closed form analytic solution is, in general, difficult to obtain, if not possible. Therefore, a numerical solution is sought. Many researchers have developed numerical technique to study the numerical solution of two point boundary value problems. Villadsen and Stewart [5] proposed solution of boundary value problem by orthogonal collocation method. Jang [6] proposed the solution of twopoint boundary value problem by the extended Adomian decomposition method. The Galerkin-finite element method is well known numerical technique for the numerical solution of differential equations. Dogan [7] proposed the Galerkinfinite element approach for the numerical solutions of Burgers' equation. Sengupta et al. [8] carried out Gakerkin finite element methods for wave problems. Kaneko et al. [9] discussed the Discontinuous Galerkin-finite element method for parabolic problems. EI-Gebeily et al. [10] studied the finite element- Galerkin method for singular self-adjoint differential equations. Sharma et al. [11] proposed Galerkinfinite Element Methods for numerical solution of advection- diffusion equation. Onah [12] proved the asymptotic convergence of the solution of a parabolic equation by using two methods namely, the Galerkin method expressed in terms of linear splines and the Finite Element Collocation method expressed by cubic spline basis functions. In this paper, we consider the following inhomogeneous second order differential equation

$$
\left\{\begin{array}{l}
u^{\prime \prime}(x)+p(x) u^{\prime}(x)+q(x) u(x)=f(x), \quad \alpha<x<\beta \\
u(\alpha)=0 \\
u(\beta)=0
\end{array}\right.
$$

where $p(x)=\mathbb{C}^{2}[\alpha, \beta] \quad p(x) \geq \lambda>0 \quad$ in $\quad[\alpha, \beta]$, $q(x)=\mathbb{C}^{1}[\alpha, \beta], q(x) \geq 0$ on $[\alpha, \beta]$ and $f(x)=\mathbb{C}^{1}[\alpha, \beta]$

We assume that problem (1.1) has a unique solution $u(x)$

In the present work, we use Galerkin-finite element method for the numerical solution of two point boundary value problems. The approach is simple and effective.

The remaining part of the article is organised as follows. In Section 2, we shall first reformulate (1.1) as a variational problem in the space variables $x$.We shall then define a Galerkin approximation $u(x)$ to the solution of (1.1) by requiring that $u$ lie in a finite-dimensional space of functions, also an error estimate is given. The Full Discretized system arising from either of the spatial discretisations is given in Section 3. In section 4 of this paper, we shall make some direct applications of approximation theory to some test 
problems. Finally, Section 5 concludes the article with final remarks

\section{Formulation of the Variational Problem and Galerkin Approximations}

This problem may also be stated in weak form: find $u \in H_{0}^{1}([\alpha, \beta])$ such that

$$
\Theta(u, w)=(f, w) \text { for } w \in H_{0}^{1}([\alpha, \beta])
$$

where

$$
\Theta(u, w)=\int_{\alpha}^{\beta}\left(-\frac{d w}{d x} \frac{d u}{d x}+w p(x) \frac{d u}{d x}+w q(x) u\right) d x,\langle u, w\rangle=\int_{\alpha}^{\beta} u w d x
$$

The (standard) Galerkin method for approximating the solution $u$ of (2.1) amounts to constructing a family of finitedimensional subspaces $\left\{S_{h}\right\} \quad 0<h<1$, and seeking $u_{h} \in S_{h}$ satisfying the linear system of equations

$$
\Theta\left(u_{h}, \chi\right)=(f, \chi) \text { for } \chi \in S_{h}
$$

We shall assume that the data are such that the unique solution $u$ of (2.1) belongs to $u \in\left(H_{0}^{1}(\Omega) \cap H^{2}(\Omega)\right)$ and satisfies the elliptic regularity estimate that for some $C>0$, independent of $f$ and $u$ we have

$$
\|u\|_{2} \leq C\|f\|
$$

Under our hypotheses, a unique solution $u_{h}$ of (2.2) exists and satisfies

$$
\left\|u-u_{h}\right\|_{1} \leq C_{\inf _{x \in S_{h}}}\|u-\chi\|_{1}
$$

for some constant $C$ independent of $h$. The existenceuniquenss of $u_{h} \in S_{h}$ is guaranteed by Lax-Milgram theorem applied to the Hilbert space $\left(S_{h},\|\cdot\|\right)$ the proof of LaxMilgram theorem is given in Appendix A. Assuming that

$$
\inf _{x \in S_{h}}\left\{\|\varphi-\chi\|+h\|\varphi-\chi\|_{1}\right\} \leq C h^{2}\|\varphi\|_{2} \quad \varphi \in\left(H_{0}^{1}(\Omega) \cap H^{2}(\Omega)\right)
$$

we obtain from (2.4) the optimal-rate $H^{1}(\Omega)$-error estimate

$$
\left\|u-u_{h}\right\|_{1} \leq C h\|u\|_{2}
$$

The $L^{2}$-error estimate is obtained by the "Nitsche trick", by letting $e=u-u_{h}$ and considering $w \in\left(H_{0}^{1}(\Omega) \cap H^{2}(\Omega)\right)$ the solution of the problem

$$
\Theta(w, \varphi)=(e, \varphi) \text { for } \varphi \in H_{0}^{1}(\Omega)
$$

Then $\|e\|^{2}=(e, e)=\Theta(w, e)=\Theta(e, w)=\Theta(e, w-\chi)$ for any $\chi \in S_{h}$,

By the continuity of $L$ in $H^{1}(\Omega) * H^{1}(\Omega)$ we have then

$$
\|e\|^{2}=C\|e\|_{1}\|w-\chi\|_{1} \leq C\|e\|_{1} h\|w\|_{2} \leq C h\|e\|_{1}\|e\| .
$$

Hence $\|e\| \leq C h\|e\|_{1} \leq C h^{2}\|u\|_{2}$

In general, assuming that for some integer $r \geq 2$

$$
\inf _{x \in S_{h}}\left\{\|w-\chi\|+h\|w-\chi\|_{1}\right\} \leq C h^{r}\|w\|_{r} \text { for } w \in\left(H_{0}^{1}(\Omega) \cap H^{r}(\Omega)\right)
$$

where $\|$.$\| denotes the norm in H^{s}(\Omega),\|\|=.\|.\|_{0}$ and the Nitsche argument give $\|e(t)\|+h\|e(t)\|_{1} \leq C h^{r}\|u\|_{r}$ $u \in\left(H_{0}^{1}(\Omega) \cap H^{r}(\Omega)\right)$

\section{Fully Discretized Finite Element Models}

We shall approximate the solution of (2.2) by requiring that $u$ and $\chi$ lie in $\left\{S_{h}\right\} \quad 0<h<1$. Let $\chi_{\kappa} \in S_{h}$ for $\kappa=1,2, \ldots, N$. Assume that the set $\chi_{1}, \ldots, \chi_{N}$ is linearly independent. Denote by $\Upsilon$ the subspace spanned by $\chi_{1}, \ldots, \chi_{N}$,let $\left\{\chi_{\kappa}\right\}_{\kappa=1}^{N}$ be a basis of $S_{h}$ where $N=\operatorname{dim} S_{h}$.We shall approximate $u$ of (2.1) by a function

$$
\begin{gathered}
u_{h}(x)=\sum_{\kappa=1}^{N} c_{\kappa} \chi_{\kappa}(x) \\
\Theta\left(u_{h}, \chi\right)=(f, \chi) \text { for } \chi \in H_{0}^{1}(\Omega) \quad \chi \in S_{h}
\end{gathered}
$$

Substituting this expression for $u_{h}$ in (2.2) and taking $\chi=\chi_{\kappa}, \kappa=1, \ldots, N$ we see that

$$
G c=f_{h}
$$

Where $G$ is the $N x N$ matrix defined by $G=G_{\kappa j}=\Theta\left(\chi_{\kappa}, \chi_{j}\right)=\int_{\alpha}^{\beta}\left(\chi_{\kappa}^{\prime} \chi^{\prime}+p(x) \chi_{\kappa} \chi_{j}^{\prime}+q(x) \chi_{\kappa} \chi_{j}\right) d x$, $1 \leq \kappa, j \leq N \quad C=\left[c_{1}, \ldots, c_{N}\right]^{T}$ and $f_{h}=\left[\left(f, \chi_{1}\right), \ldots,\left(f, \chi_{N}\right)\right]^{T}$. Where $G$ is a positive definite matrice.

\section{Numerical Example}

In this section, some numerical examples are studied to demonstrate the accuracy of the present method. The examples are computed using MatlabR2012b. The versatility and accuracy of proposed method is measured using $L_{\infty}$.

$$
L_{\infty}=\left\|u-U_{N}\right\|_{\infty}=\max _{j}\left|u_{j}-\left(U_{N}\right)_{j}\right| \mid
$$

Example 1. Considering equation 
$u^{\prime \prime}(x)+p(x) u^{\prime}(x)+q(x) u(x)=f(x), \alpha=0 \leq x \leq \beta=1$

with boundary conditions

$$
\begin{aligned}
& u(0)=0 \\
& u(1)=0
\end{aligned}
$$

where the function $p(x)$ and $q(x)$ are assumed constant, $-3,2$ respectively, while the function $f(x)$ is assumed 1 .

The true solution of this problem is $u(x)=c_{1} e^{2 x}+c_{2} e^{x}+\frac{1}{2}$, where $c_{1}=\frac{1 / 2}{\exp (1)}, c_{2}=-\frac{1}{2}\left(1+\frac{1}{\exp (1)}\right)$

Table 1. \|\|$_{\infty}$ concentration errors. Linear elements

\begin{tabular}{ll}
\hline Elements & $\|L\|_{\infty}$ \\
\hline 10 & $4.5593 \mathrm{E}-004$ \\
40 & $3.0706 \mathrm{E}-005$ \\
100 & $4.9871 \mathrm{E}-006$ \\
\hline
\end{tabular}

Example 2. Let's consider the same example with mixed boundary conditions as below

$$
\begin{aligned}
& u(0)=0 \\
& u^{\prime}(1)=1
\end{aligned}
$$

The true solution of this problem is $u(x)=c_{1} e^{2 x}+c_{2} e^{x}+\frac{1}{2}$, where $c_{1}=\frac{\left(1+\frac{1}{2} \exp (1)\right)}{(2 \exp (2)-\exp (1))}, c_{2}=\frac{-(1+\exp (2))}{(2 \exp (2)-\exp (1))}$

Table 2. \|\|$_{\infty}$ concentration errors. Linear elements

\begin{tabular}{ll}
\hline Elements & $\|L\|_{\infty}$ \\
\hline 10 & $3.7646 \mathrm{E}-004$ \\
40 & $2.2106 \mathrm{E}-005$ \\
100 & $3.5089 \mathrm{E}-006$ \\
\hline
\end{tabular}

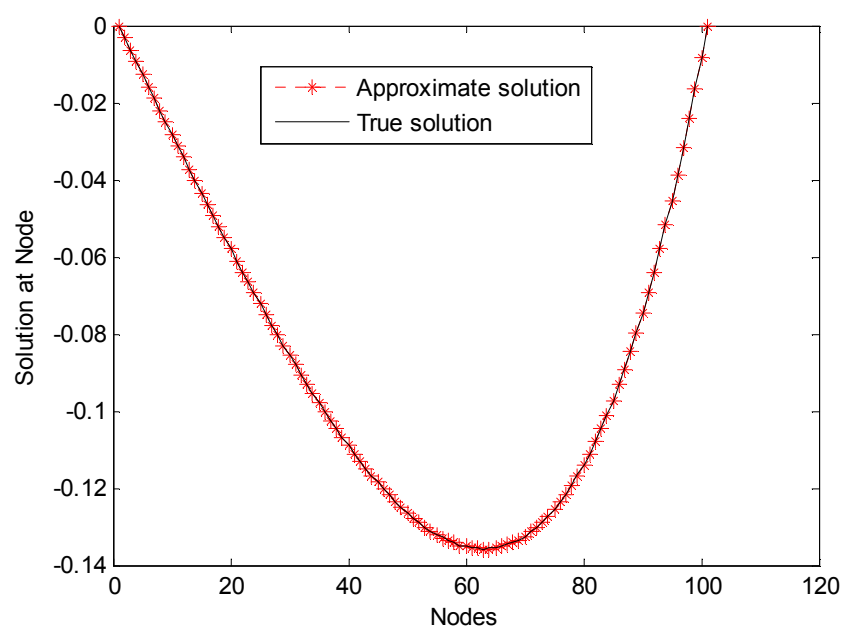

Fig. 1. Comparison of numerical and exact solution of Example 1. Linear elements

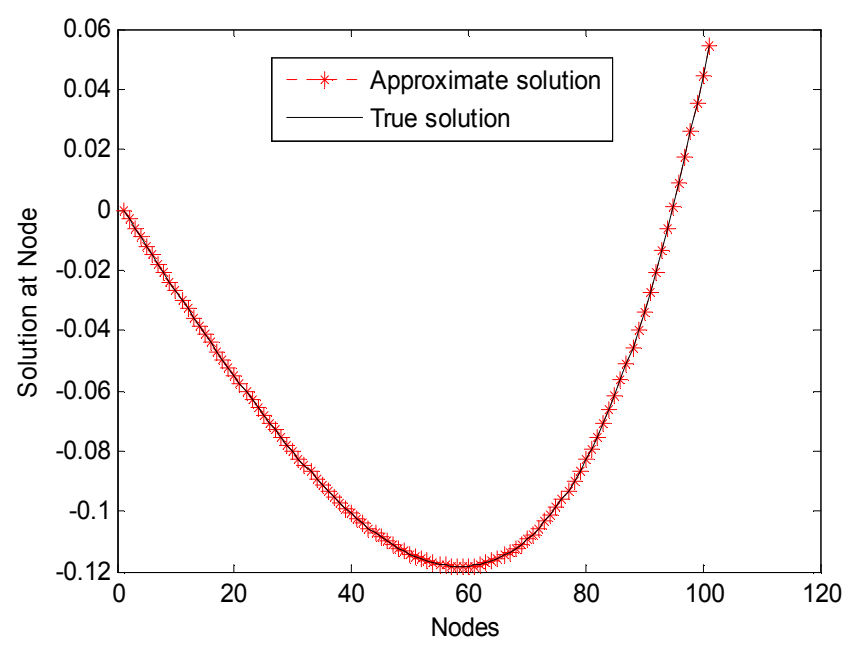

Fig. 2. Comparison of numerical and exact solution of Example 2. Linear elements

Example 3. Considering equation

$$
x^{2} u^{\prime \prime}(x)-2 x u^{\prime}(x)+4 u(x)=x^{2} \quad 10 \leq x \leq 20
$$

with boundary conditions

$$
\begin{aligned}
& u(10)=0 \\
& u(20)=100
\end{aligned}
$$

The true solution of this problem is $0.00102 x^{4}-0.16667 x^{2}+64.5187 \frac{1}{x}$.

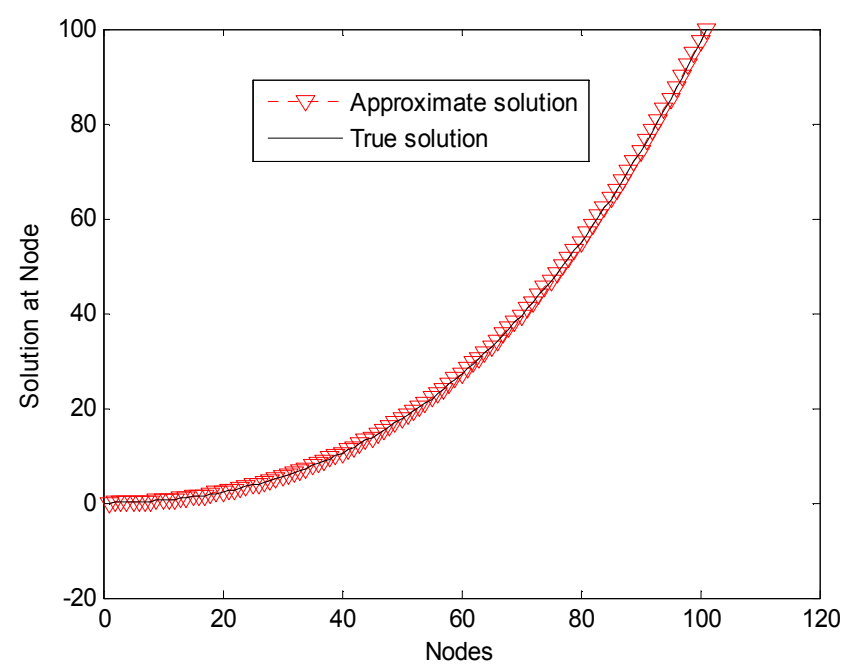

Fig. 3. Comparison of numerical and exact solution of Example 3. Linear elements

Table 3. \|\|$_{\infty}$ concentration errors. Linear elements

\begin{tabular}{ll}
\hline Elements & $\|L\|_{\infty}$ \\
\hline 20 & $6.8 \mathrm{E}-2$ \\
40 & $5.02 \mathrm{E}-2$ \\
100 & $1.02 \mathrm{E}-2$ \\
\hline
\end{tabular}




\section{Concluding Remarks}

In this article, Galerkin-finite element method is proposed to find the approximate solutions of two point boundary value problems. In the solution procedure, the first step is to make weak formulation and then develop finite element formulation. Lastly, weighted average is used for fully discretization. As test problem, three different solutions of three point boundary value problems are chosen. Also, a comparison of numerical and analytical solutions is made and found that the proposed scheme has good accuracy.

\section{Appendix A}

Theorem 1 (Lax-Milgram Theorem). Let $H$ be a (real) Hilbert space and let $\Theta(\cdot, \cdot): H \times H \rightarrow \mathbb{R}$ be a bilinear form on $H$ which satisfies:

$$
\begin{aligned}
& \text { 1. }|\Theta(\phi, \psi)| \leq c_{1}\|\phi\|\|\psi\| \quad \forall \phi, \psi \in H \\
& \text { 2. } \Theta(\phi, \phi) \geq c_{2}\|\phi\|^{2} \quad \forall \phi \in H
\end{aligned}
$$

where $c_{1}, c_{2}$ are positive constants independent of $\phi, \psi \in H$.

Let $F: H \rightarrow \mathbb{R}$ be a (real valued) linear functional on $H$ such that.

3. $\exists c_{3}>0 \forall \psi \in H|F(\psi)|<c_{3}|\psi|$

Then there exists a unique $u \in H$ satisfying

$$
\Theta(u, w)=F(w) \quad \forall w \in H
$$

Moreover,

$$
\|u\| \leq \frac{1}{c_{2}}\|F\|
$$

Proof. Let $\phi \in H$ be fixed. Then $\Phi: H \rightarrow \mathbb{R}$ defined for every $w \in H$ by $\Phi(w)=\Theta(\phi, w)$ defines a continuous linear functional on $\mathrm{H}$. For boundedness observe that for each $w \in H$

$$
|\Phi(w)|=|\Theta(\phi, w)| \leq c_{1}\|\phi\|\|\psi\|
$$

Hence $\|\Phi\| \leq c_{1}\|\phi\|<\infty$

By the Riesz Representation Theorem therefore, there exists a unique element $\widehat{\phi}$ such that

$$
\Phi(w)=\Theta(\phi, w)=(w, \widehat{\phi}) \forall w \in H
$$

Hence for every $\phi \in H$ we define a $\bar{\phi} \in H$ by (A.1) and denote the correspondence $\varphi \mapsto \widehat{\phi}$ by $\widehat{\phi}=\Lambda \phi$

$$
\Theta(\phi, w)=(w, \Lambda \phi) \quad \forall w \in H \quad \forall \phi \in H
$$

Now $\Lambda$ is a linear operator defined on $H$. We claim now that $\Lambda$, defined by (A.2) has a range $\operatorname{Ran}(\Lambda)$ which is a closed subspace of $H$. Let $\widehat{\phi}_{n}=\Lambda \phi_{n}$ be a convergent sequence, such that $\widehat{\phi}_{n} \mapsto \widehat{\phi}$. Now, since $\Theta\left(\phi_{n}, w\right)=\left(w, \Lambda \phi_{n}\right)$ $\forall w \in H \Rightarrow \Theta\left(\phi_{n}-\phi_{m}, w\right)=\left(\Lambda \phi_{n}-\Lambda \phi_{m}, w\right) \quad \forall w \in H$. Choose $w=\phi_{n}-\phi_{m}$ and using (2) get $\left\|\phi_{n}-\phi_{m}\right\| \leq \frac{1}{c_{2}}\left\|\Lambda \phi_{n}-\Lambda \phi_{m}\right\|$. Hence $\left\{\phi_{n}\right\}$ is a Cauchy sequence in $H$, there exist $\phi \in H$ such that $\phi_{n} \mapsto \phi$. We now show that $\widehat{\phi}=\Lambda \phi$ thus showing that $\widehat{\phi} \in \operatorname{Ran}(\Lambda)$, that $\operatorname{Ran}(\Lambda)$ is closed.

Now $\left|\Theta\left(\phi_{n}, w\right)-\Theta(\phi, w)\right| \leq C_{1}\left\|\phi_{n}-\phi\right\|\|w\| \quad \forall w \in H$ gives that

$$
\lim _{n \rightarrow \infty} \Theta\left(\phi_{n}, w\right)=\Theta(\phi, w) \forall w \in H
$$

Also

$$
\Lambda\left(\phi_{n}, w\right)=\left(\widehat{\phi}_{n}, w\right) \rightarrow(\widehat{\phi}, w)
$$
since

$\left|\left(\widehat{\phi}_{n}, w\right)-(\widehat{\phi}, w)\right| \leq\left\|\widehat{\phi}_{n}-\widehat{\phi}\right\|\|w\|$. Since $\Theta\left(\phi_{n}, w\right)=\Lambda\left(\phi_{n}, w\right)$ $\forall w \in H \Rightarrow \Theta(\phi, w)=(\widehat{\phi}, w)$. Hence $\operatorname{Ran}(\Lambda)$ is closed. Also we claim that $\operatorname{Ran}(\Lambda)=H$

Given $F$ on $H$, by Riesz representation $\exists ! \xi \in H$ such that $F(w)=(\xi, w) \forall v \in H$.Since $\operatorname{Ran}(\Lambda)=H \quad \exists u \in H$ such that $\Lambda u=\xi$.Hence $\exists u$ such that

$$
F(w)=(\Lambda u, w)=\Theta(u, w) \forall w \in H
$$

For uniqueness, suppose that $\exists u_{1} \neq u_{2}$ such that $\Theta\left(u_{1}, w\right)=F(w)=\Theta\left(u_{2}, w\right) \quad \forall w \in H$. Hence $\Theta\left(u_{1}-u_{2}, w\right)=0$ $\forall w \in H \Rightarrow \Theta\left(u_{1}-u_{2}, u_{1}-u_{2}\right) \geq c_{2}\left\|u_{1}-u_{2}\right\|^{2} \Rightarrow u_{1}=u_{2}$

Since $\Theta(u, u)=F(u),(1),(2)$ give that $u \neq 0 \quad c_{2}\|u\| \leq|F(u)|$ from which $\|u\| \leq \frac{1}{c_{2}} \frac{|F(u)|}{\|u\|}$. Hence

$$
\|u\| \leq \sup _{w \neq 0} \frac{1}{c_{2}} \frac{|F(w)|}{\|w\|}=\frac{1}{c_{2}}\|F\|
$$

\section{References}

[1] C.w. Cryer, The numerical solution of boundary value problems for second order functional differential equations by finite differences, Numer. Math. 20 (1973) 288-299.

[2] Y.F. Holt, Numerical solution of nonlinear two-point boundary value problems by finite difference method, Comm. ACM 7 (1964) 363-373.

[3] P.G. Ciarlet, M.H. Schultz and R.S. Varga, Numerical methods of high order accuracy for nonlinear boundary value problems, Numer. Math. 13 (1967) 51-77.

[4] S Arora, S S Dhaliwal, V K Kukreja. Solution of two point boundary value problems using orthogonal collocation on finite elements. Appl. Math. Comput., 171(2005):358-370.

[5] J Villadsen, W E Stewart. Solution of boundary value problems by orthogonal collocation. Chem. Eng. Sci., 22(1967): 1483-1501. 
[6] B Jang. Two-point boundary value problems by the extended Adomian decomposition method. J. Comput. Appl.Math., 219 (1)(2008): 253-262.

[7] A Dogan. A Galerkin finite element approach of Burgers' equation. Appl. Math. Comput., 157 (2)(2004): 331-346.

[8] T K Sengupta, S B Talla, S C Pradhan. Galerkin finite element methods for wave problems. Sadhana, 30 (5)(2005): 611-623.

[9] H Kaneko, K S Bey, G J W Hou. Discontinuous Galerkin finite element method for parabolic problems. Appl. Math. Comput., 182 (1)(2006):388-402.

[10] M A EI-Gebeily, K M Furati, D O'Regan. The finite elementGalerkin method for singular self-adjoint differential equations. J. Comput. Appl. Math., 223 (2)(2009): 735-752.
[11] D Sharma, R Jiwari, S Kumar. Galerkin-finite Element Methods for Numerical Solution of Advection- Diffusion Equation. Int. J. Pure and Appl. Math., 70 (3)(2011): 389-399.

[12] S E Onath. Asymptotic behavior of the Galerkin and the finite element collocation methods for a parabolic equation. Appl. Math. Comput., 127(2002):207-213.

[13] T Jangveladze, Z Kiguradze, B Neta. Galerkin finite element method for one nonlinear integro-differential model. Appl. Math. Comput., 217 (16)(2011):6883-6892. 